\title{
SPring-8 Beamlines for High Pressure Science with Multi-Anvil Apparatus
}

\author{
W. Utsumi, K.Funakoshi*, S.Urakawa**, M.Yamakata, K.Tsuji***, H.Konishi, and O.Shimomura \\ Japan Atomic Energy Research Institute, Kamigori, Ako-gun, Hyogo 678-12, Japan \\ *Japan Synchrotron Radiation Research Institute, Kamigori, Ako-gun, Hyogo 678-12, Japan \\ **Okayama University, Tsushima-naka, Okayama 700, Japan \\ ***Keio University, Hiyoshi, Kohoku-ku, Yokohama, Kanagawa 223, Japan
}

\begin{abstract}
The SPring- 8 will open for public use in October, 1997. Three beamlines are now under construction for high pressure research with large volume high pressure apparatus. Two multi-anvil apparatus will be installed on these beamlines. One is a 1500 ton press with a big DIA type guide-block for two stage compression, which is used for the energy dispersive $x$-ray diffraction study. The other is a 180 ton small DIA press similar to MAX90 at the Photon Factory, which is used for both energy and angle dispersive diffraction as well as an x-ray absorption study.

[SPring-8, multi-anvil apparatus, synchrotron radiation, $x$-ray diffraction]
\end{abstract}

\section{Introduction}

SPring-8, a third-generation synchrotron radiation facility, is now being constructed at Harima Science Garden City in Hyogo prefecture in Japan, under the JAERI -RIKEN SPring- 8 Project Team, organized by the Japan Atomic Energy Research Institute (JAERI) and the Institute of Physical and Chemical Research (RIKEN). SPring- 8 is an accelerator complex consisting of a $1 \mathrm{GeV}$ linac, an $8 \mathrm{GeV}$ synchrotron, and an $8 \mathrm{GeV}$ storage ring. The storage ring operates at an energy of $8 \mathrm{GeV}$ with an expecte. lifetime of about 50 hours at a beam current of $100 \mathrm{~mA}$. 38 beamlines with insertion devices and 23 beamlines from bending magnets are planned to be installed in this storage ring.

The first synchrotron radiation from the storage ring was achieved in March of 1997. In parallel with the construction of the accelerators, ten public beamlines are being built by the recommendation of the Beamline Advisory Committee. These ten public beamlines will open for public use in October, 1997. Also under way in parallel with the public beamlines, is the construction of three JAERI beamlines and three RIKEN beamlines, which are built for their own use.

The great success of high pressure research combined with synchrotron radiation at many second-generation synchrotron radiation facilities in the world stimulated the Japanese high pressure scientists, and they proposed several SPring-8 beamlines for high pressure research to the Beamline Advisory Committee. This proposal was accepted and two public beamlines were allowed to be built for high pressure science; one is for the use of a large volume press (BL04B1), and the other is for diamond anvil cells (BL10XU). After much discussion, the high pressure users group has decided to install a multi-anvil type high pressure apparatus on the BL04B1 beamline, which is a 1500 ton press with a DIA type guideblock with two stage compression. This press system has been already operated and the first preliminary experiment with synchrotron radiation beam was carried out on July of 1997.

In addition to the BL04B 1 public beamline, two JAERI beamlines (BL14B1 and BL11XU) are also available for high pressure research with multi-anvil apparatus. JAERI staff members are designing a small multi-anvil press for these beamlines. It is a 180 ton small DIA press and can be used for both energy dispersive and angle dispersive $x$-ray diffraction studies as well as absorption experiments, and it can be easily moved between the two beamlines.

Here, we report these three SPring- 8 beamlines for high pressure science with multi-anvil apparatus. The layout of the beamlines at the SPring- 8 is shown in Fig.1.

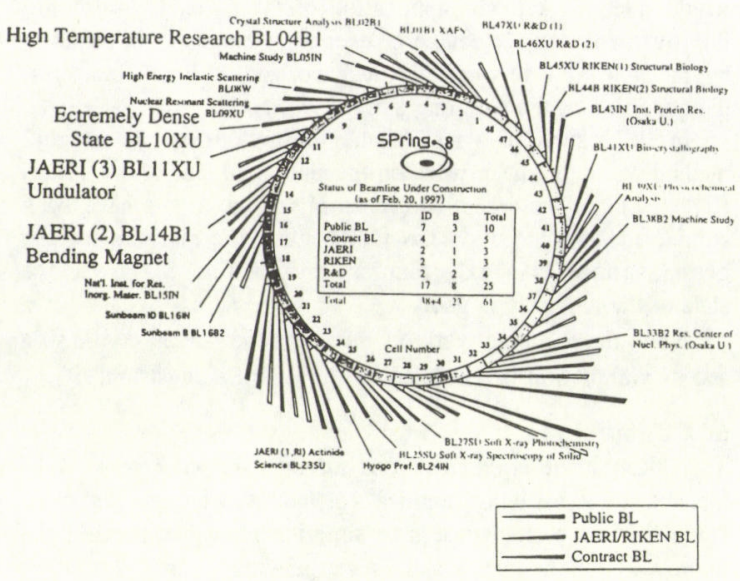

Fig.1. Beamline layout at the SPring-8. 


\section{High Temperature Beamline (BL04B1)}

BL04B1, one of the public beamlines, is called the "High Temperature Research Beamline." It is a bending magnet beamline. This beamline has no monochromators or focusing mirrors and thus only white $\mathrm{x}$-rays $(10-150 \mathrm{keV})$ are available in the experimental hutches. Two experimental stations are built in tandem; one is for the multi-anvil apparatus, and the other is for a high pressure gas vessel using helium gas as a pressure medium. (Detail about this high pressure gas apparatus is described by Tamura et al. in this volume.)

In the upstream experimental station, a large volume multianvil type high pressure apparatus is installed (Fig.2). This apparatus consists of a 1500 ton press, a guide-block, vertical and horizontal goniometers, positioning stages, and their computer control facilities.

The main press has a 1500 ton ram-force uniaxial press with four supporting posts. A big DIA type guide-block is mounted on the press. Pressure generation is made by the twostage compression method (so-called 6-8 system), where the first stage is the six anvils mounted on the DIA guide-block and the second stage consists of eight cubic anvils with truncated edges. An octahedral shaped pressure medium is compressed by the eight inner anvils, which are compressed by the outer six anvils. Either tungsten carbide or polycrystalline sintered diamonds can be used for the second stage anvils. This system has a capability of generating pressures up to $25 \mathrm{GPa}$ and a temperature of $3000 \mathrm{~K}$ in a large sample volume $\left(1 \mathrm{~mm}^{3}\right)$ by using tungsten carbide anvils. Pressure generation higher than $40 \mathrm{GPa}$ is possible when anvils are replaced with sintered diamond.

For the $\mathrm{x}$-ray experiments, two single-axis goniometers (vertical and horizontal directions) are equipped. The vertical goniometer covers diffraction angles of $\pm 25 \mathrm{deg}$, which can be operated by using one sintered diamond anvil as an $\mathrm{x}$-ray transparent window (Fig.3). The angles of the horizontal goniometer is limited to $\pm 10 \mathrm{deg}$, because of the narrowness of the first-stage anvil gap. The horizontal goniometer system can be used both for sintered diamond and tungsten carbide as the second stage anvils.

The press is mounted on an $x-y-z$ translation stage with a rotation axis to align the beam with the sample. The divergence slit is mounted separately on a $y-z$ positioning stage in front of the press. The vertical goniometer is mounted on a $y-z$ positioning stage, and the horizontal one is mounted on an $x-y$ $z$ stage. Each stage is controlled by a servo or pulse motor and computer system. Every movement is monitored by a linear magnet scale and its signal is fed back to the computer to align the stage precisely. With this control system, all the stages can be controlled within an accuracy of 1 micron. Since this high pressure system is fixed in this beamline and only white $x$-rays are available, most experiments are related to powder $x$-ray diffraction by the energy dispersive method. A Ge-SSD is used for the detector system.

The construction of this beamline and experimental stations have been completed. The high pressre apparatus is already installed in the experimental hutch, and system is ready to operate. On July 7th, 1997, the main shutter was opened for the first time, and the first beam of this beamline was observed. After the $\mathrm{x}$-ray leakage test of the hutch, a preliminary high pressure experiment was carried out using the synchrotron radiation beam to check out the system performance. This beamline will open for public use in October, 1997. More than ten experimental proposals have been subitted for the first halfyear beamtime period (from October 1997 to March 1998). During this first half year, the storage ring will be operated at a beam current of $20 \mathrm{~mA} .100 \mathrm{~mA}$ operation will be start later than April of 1998.
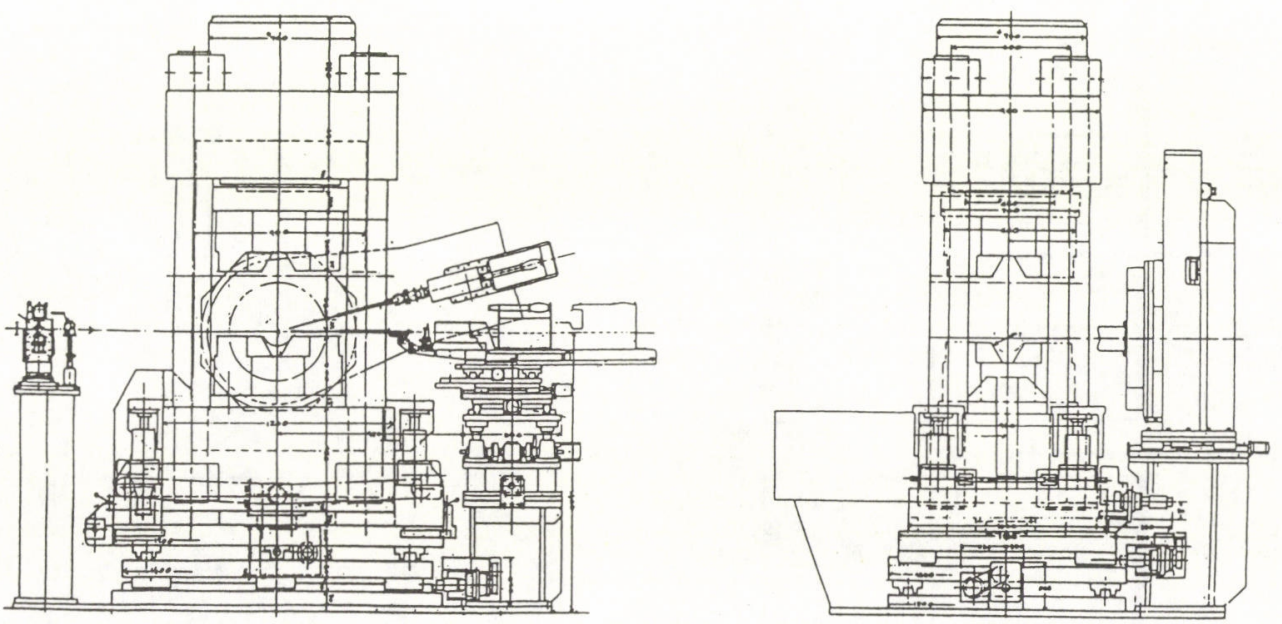

Fig.2. 1500 ton multi-anvil apparatus installed at the BL04BI beamline. 


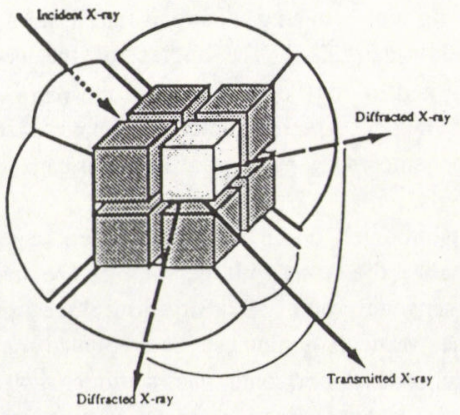

Fig.3. X-ray path in the two stage compression system.

\section{JAERI Beamlines (BL14B1, BL11XU)}

JAERI (Japan Atomic Energy Research Institute) is planning to build three beamlines at the SPring- 8 for its own use. Two of those are hard $x$-ray beamlines and one is for soft $\mathrm{x}$-ray experiments.

BL14B1, one of the JAERI hard $x$-ray beamlines, is a bending magnet beamline. It is designed for various diffraction and absorption experiments using monochromatized or white $x$-rays. This beamline has two experimental hutches in tandem, and its transport channel contains two mirrors and a fixed-exit double crystal monochromator. Three different experimental modes can be selected by switching the optics (Fig4). In the white $x$-ray mode (A), all optics are removed from the direct beam so that the white beam is available in the upstream white $\mathrm{x}$-ray hutch. Monochromatized $\mathrm{x}$-rays are also available in the same hutch (B). An adjustable inclined geometry of the monochromator covers a wide $x$-ray energy range (3.5-90keV) using a single pair of silicon crystals. When focused monochromatized $\mathrm{x}$-rays $(3.5-20 \mathrm{keV})$ are needed, two mirrors are used (C). X-rays made parallel by the first mirror enter the monochromator, and the second
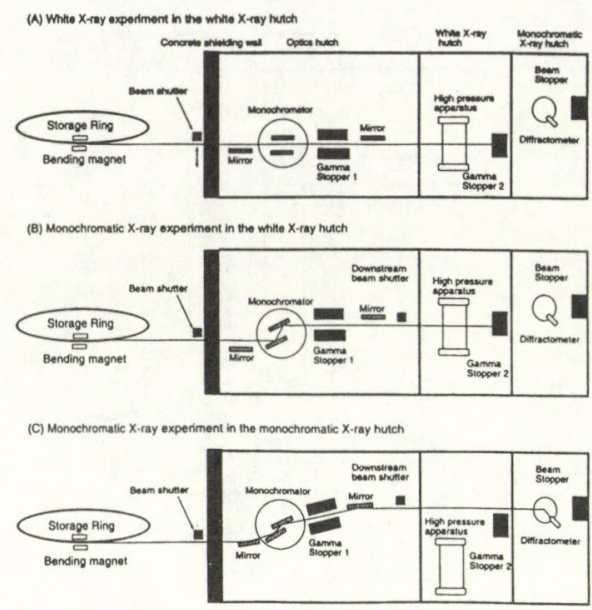

Fig.4. Three selective optics modes at BL14BI beamline. monochromator crystal and the second mirror focus the beam in the horizontal and vertical direction, respectively.

Another JAERI hard $\mathrm{x}$-ray beamline is an undulator beamline (BL11XU). X-rays from a SPring- 8 standard in-vacuum undulator are monochromatized by another inclined double crystal monochromator. Beamline design is now in progress.

For these JAERI beamlines, a new multi-anvil system is being designed. Fig.5 is a schematic drawing of the system. A 180 ton DIA type multi-anvil press is chosen as a high pressure press. It is similar to MAX90 at the Photon Factory in Tsukuba, Japan. A cubic shaped pressure medium is compressed by the six single-stage anvils. Although the pressure region is limited to about $15 \mathrm{GPa}$ using tungsten carbide anvils, this press is much more compact than that on BL04B1. This system is characterized by its optics. In order to carry out an angle dispersive powder diffraction experiment as well as an energy dispersive diffraction study, it has two-dimensional detectors such as an Imaging Plate or a CCD camera in addition to a Ge-SSD. Two concentric vertical goniometers are placed by the high pressure apparatus. An SSD for energy dispersive diffraction is mounted on the outer goniometer. On the inner goniometer, a multi-channel collimator (soller slits) is mounted. This multichannel collimator is to reduce the background noise from the sample surrounding materials such as the pressure medium for the angle dispersive experiments. A center slit of the multi-channel collimator is used also as a single collimator in the energy dispersive experimental mode. X-ray absorption experiment under high pressure can be carried out using a monochromatized beam too.

The construction of BL14B1 beamline will be completed at the end of October of 1997, and the new high pressure system is planed to be installed in its white x-ray hutch by March of 1998 . The construction of BL11XU has just started. We hope we can start experiments using its brighter undulator beam in the fall season of 1998 .

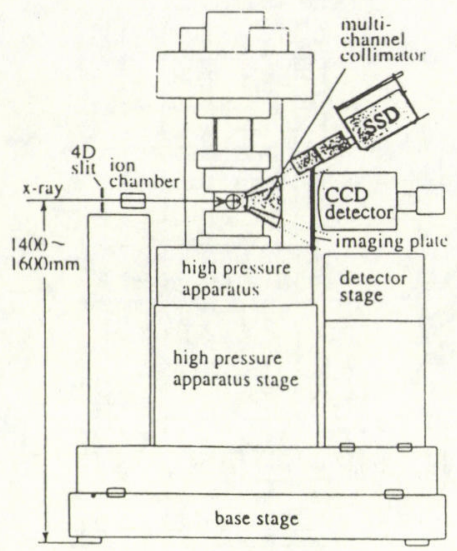

Fig.5. Schematic drawing of the high pressure press and optical system installed at JAERI beamline. 